\title{
Synthesis of high-density arrays of graphene nanoribbons by anisotropic metal-assisted etching
}

Hiroki Ago, ${ }^{* a, b, c}$ Yasumichi Kayo, ${ }^{\mathrm{b}}$ Pablo Solís-Fernández, ${ }^{\mathrm{a}}$ Kazuma, Yoshida, ${ }^{\mathrm{b}}$ Masaharu Tsuji ${ }^{\mathrm{a}, \mathrm{b}}$

a Institute for Materials Chemistry and Engineering, Kyushu University, Fukuoka 816-8580, Japan

${ }^{\mathrm{b}}$ Graduate School of Engineering Sciences, Kyushu University, Fukuoka 816-8580, Japan

${ }^{\mathrm{c}}$ PRESTO, Japan Science and Technology Agency (JST), Saitama 332-0012, Japan

\begin{abstract}
We demonstrate the synthesis of highly aligned dense arrays of graphene nanoribbons (GNRs) based on metal-catalyzed etching of chemical vapor deposition-grown single-layer graphene. In order to obtain GNR arrays, controlling the direction of the etching becomes an important task. Crystalline surfaces of $\mathrm{r}$ - and a-planes of sapphire $\left(\alpha-\mathrm{Al}_{2} \mathrm{O}_{3}\right)$ were found to induce anisotropic etching of the graphene along their specific crystallographic directions. In contrast, anisotropic surface of ST-cut quartz induced few etching lines. We found that the graphene etching is strongly dependent on the metal species; the order of the catalytic activity of metal nanoparticles is $\mathrm{Ni}>\mathrm{Fe}>\mathrm{Cu}$. Etching temperature and $\mathrm{H}_{2}$ concentration also strongly influenced the density and quality of the etching lines. Our anisotropic graphene etching is expected to offer a new route toward the synthesis of high density GNR array in large area without relying on any lithographic techniques for future electronic devices.
\end{abstract}

* Corresponding author: Tel: +81-92-583-7817, E-mail: ago@ cm.kyushu-u.ac.jp (H. Ago) 


\section{Introduction}

Graphene has attracted great interest due to its unique physical properties originated in its ideal two-dimensional (2D) structure and exceptionally high carrier mobility which promise applications in many electronic devices, such as field-effect transistors, high-frequency transistors, integrated circuits, and chemical/biochemical sensors [1-4]. Furthermore, optical transparency and mechanical flexibility of graphene open up the possibility of graphene-based flexible devices [5,6]. However, since graphene has no band gap, engineering of its band structure is a critical issue when one applies graphene to semiconductor devices.

Various methods to open a gap in graphene's band structure have been proposed [7], such as vertical biasing of double-layer graphene [8,9], applying mechanical strain [10], chemical functionalization [11], and quantum confinement into nanostructures [12-14]. Theoretical and experimental works have demonstrated that the quantum confinement in one-dimensional (1D) graphene structures, known as graphene nanoribbons (GNRs), can potentially open a band gap [12-15]. The band gap is inversely proportional to the width of the GNRs [14], thus making necessary to produce narrow ribbons in order to realize an appropriate band gap. Several bottom-up methods have been developed for growing GNRs on metal catalysts [1621], but throughput is still relatively low. Production of GNRs by top-down approaches benefits by the large-area single-layer graphene that is widely available by chemical vapor deposition (CVD) [22,23], thus allowing the synthesis of arrays of GNRs at large scale. Usually, lithographic techniques, such as electron-beam lithography and block copolymer or nanowire-templated lithographies, are combined with plasma oxidations to pattern graphene into 1D nanostructures [24-28]. Unzipping of carbon nanotubes by $\mathrm{O}_{2}$ plasma or strong acid treatment is another route to synthesize GNRs [29-31]. However, these top-down approaches include relatively strong oxidative treatments and inevitably damage the edges of GNRs and sometimes the GNR itself. Recently, He ion beam and neutral beam were used to pattern 
graphene [32,33], but they require special equipment. Therefore, a facile top-down method which does not rely on oxidative treatments and applicable to large-area graphene is required to synthesize massive arrays of GNRs.

Metal-assisted graphene etching is a unique method employed to pattern graphene. When certain metal nanoparticles deposited on graphene are heated in a hydrogen environment, they start to catalyze the reaction between hydrogen and the carbon atoms of the underlying graphene, releasing them as $\mathrm{CH}_{4}$ gas [34-36]. As the nanoparticles move across the graphene surface, this reaction leaves narrow etched lines in the graphene whose width nearly corresponds to the nanoparticle diameter [37,38]. In the case of few layer graphene and graphite, the metal-assisted etching is known to occur mainly along zigzag directions of the graphene $[34,35,39,40]$ so that triangular structures are frequently observed after the etching. Recently, r-plane single-crystalline sapphire $\left(\alpha-\mathrm{Al}_{2} \mathrm{O}_{3}\right)$ was used as a substrate in order to control the direction of metal nanoparticles $[41,42]$. The anisotropic surface of the sapphire substrate guides the nanoparticles to move parallel to a specific crystallographic direction of the sapphire. This is effective especially for single-layer graphene, because metal nanoparticles are more strongly influenced by the underlying substrate than by the carbon lattice of the graphene. We note that this etching process is free from oxidation of graphene, because the reaction proceeds in a hydrogen environment and no oxygen gas is used to pattern graphene.

To synthesize narrow graphene nanoribbons by the metal-assisted etching, achieving high etching density in graphene is essential. In previous works, different metal nanoparticles were used to etch graphene and graphite [34-37,39-42]. However, there is a lack of information on the relative catalytic activity and the effects of parameters like the employed substrate, etching temperature, and $\mathrm{H}_{2}$ gas concentrations. Therefore, further study is necessary to understand this metal-assisted etching phenomenon as well as to achieve high etching densities. Here, we 
systematically investigated the anisotropic etching of large-area CVD graphene in terms of the influence of crystalline substrates, metal species, etching temperatures, and gas atmospheres. We found that under certain experimental conditions, Ni nanoparticles on top of r-plane sapphire can produce large areas of aligned GNR arrays with average width of $25 \mathrm{~nm}$. Our results can be applied to large scale production of dense and highly aligned GNR arrays that can be developed to future carbon-based electronics.

\section{Experimental}

\subsection{Growth of graphene and transfer process}

Large-area single-layer graphene was synthesized by CVD on a heteroepitaxial $\mathrm{Cu}(111)$ thin film [43-45]. The crystalline $\mathrm{Cu}$ film catalyzes the growth of high quality graphene at high temperature $\left(1075^{\circ} \mathrm{C}\right)$ under the flow of $\mathrm{CH}_{4}, \mathrm{H}_{2}$, and $\mathrm{Ar}$ gas mixture. The domain size of CVD graphene used in our experiments is typically 10-50 $\mu \mathrm{m}$. The as-grown graphene is easily transferrable to other substrates due to the flat and rigid supporting sapphire substrate.

For the graphene transfer, a poly(methyl methacrylate) (PMMA) thin film was spin coated over the as-grown graphene, followed by chemical etching of the $\mathrm{Cu}$ in a $0.5 \mathrm{M} \mathrm{FeCl}_{3}$ aqueous solution [44]. The PMMA/graphene stack was carefully washed with Milli-Q water to remove the residual $\mathrm{FeCl}_{3}$ etchant and then transferred onto the substrate used for the metal assisted etching. In our case, four types of single-crystalline substrates, sapphire r-plane $(\alpha-$ $\left.\mathrm{Al}_{2} \mathrm{O}_{3}(1102)\right)$, sapphire a-plane(1100), and ST-cut quartz $\left(\mathrm{SiO}_{2}\right)$, and a Si substrate with a 300 nm-thick amorphous oxide layer $\left(\mathrm{SiO}_{2} / \mathrm{Si}\right)$, were studied. Finally, the PMMA was removed with hot acetone. The transfer of single-layer graphene was confirmed by Raman spectroscopy (Tokyo Instruments, Nanofinder30) and scanning electron microscope (SEM, 
Hitachi S-4800). As the employed substrates are transparent, identification of the graphene by optical microscopy was not performed.

\subsection{Metal-assisted etching}

Metal nanoparticles, which will act as catalyst for the etching reaction of graphene, were deposited on the transferred graphene. These metal nanoparticles were deposited by radiofrequency $(\mathrm{RF})$ magnetron sputtering at room temperature in $\operatorname{Ar}$ gas (0.6 Pa), using a Shibaura Mechatronics CFS-4ES. In this work, we investigated the catalytic activity of three different metal nanoparticles, $\mathrm{Ni}, \mathrm{Fe}$, and $\mathrm{Cu}$ nanoparticles. The sputtering condition was tuned so as to obtain nanoparticles suitable for metal-assisted graphene etching (sputter power $100 \mathrm{~W}$, typical deposition time 60-120 seconds). The graphene with the deposited metal nanoparticles was then submitted to an annealing procedure in two stages under $\mathrm{Ar} / \mathrm{H}_{2}$ gas flow. First, it was annealed at $400{ }^{\circ} \mathrm{C}$ for one hour, in order to remove impurities such as PMMA residue from the graphene surface. The second stage was the metal-assisted etching itself, and was carried out at temperatures in the range of $1000-1100{ }^{\circ} \mathrm{C}$. After 30 minutes of etching, the sample was cooled down to room temperature under the same gas flow. In order to minimize the thermal agglomeration of the metal nanoparticles, the sample was set outside of the hot zone during the periods when the furnace temperature was increased. After the etching procedure, the samples were studied by SEM, atomic force microscope (AFM, Bruker Nanoscope V), Raman spectroscope with $532 \mathrm{~nm}$ excitation. For the carrier transport measurements, field-effect transistors (FETs) were fabricated with arrays of GNRs transferred onto a $\mathrm{SiO}_{2} / \mathrm{Si}$ substrate and measured by a semiconductor analyzer (Agilent B1500A) in vacuum $\left(10^{-4} \mathrm{~Pa}\right)$. The GNR array was re-transferred from sapphire to $\mathrm{SiO}_{2} / \mathrm{Si}$ by using a PMMA film and $\mathrm{KOH}$ etching solution. 


\section{Results and discussion}

\subsection{Metal-assisted etching of graphene on various substrates}

Figure 1a schematically shows the steps involved in our metal-assisted graphene etching. Large-area single-layer graphene is grown by ambient pressure CVD on a heteroepitaxial $\mathrm{Cu}(111)$ film and then transferred onto four different substrates (r-, a-plane sapphire, ST-cut quartz, and $\mathrm{SiO}_{2} / \mathrm{Si}$ substrates). The catalytic etching of graphene is expressed as:

$$
\mathrm{C}(\text { graphene })+2 \mathrm{H}_{2} \rightarrow \mathrm{CH}_{4} \quad \Delta H_{\mathrm{f}}^{\mathrm{o}}=-74.9 \mathrm{~kJ} / \mathrm{mol}
$$

where $\Delta H_{\mathrm{f}}{ }^{\mathrm{o}}$ is the standard enthalpy of formation. This etching reaction is exothermic and it can be considered as a reverse reaction of graphene growth. Figure $1 \mathrm{~b}-\mathrm{g}$ shows effects of the substrates on the etching density of CVD graphene. Three single crystalline substrates and a $\mathrm{Si}$ substrate with a 300 nm-thick amorphous $\mathrm{SiO}_{2}$ layer were studied. Graphene with sputtered $\mathrm{Ni}$ nanoparticles was annealed at $1100{ }^{\circ} \mathrm{C}$ in $15 \%-\mathrm{H}_{2}$ in $\mathrm{Ar}$ gas. Note that we confirmed the absence of etching lines in graphene without depositing metal nanoparticles, indicating that the $\mathrm{FeCl}_{3}$ etchant used in the transfer process was sufficiently removed and that the etching lines are exclusively produced by the sputtered catalyst.

Previous reports have shown that the nature of the employed substrate plays a fundamental role in the trajectory of the catalyst nanoparticles during the etching procedure [41,42]. The commonly employed amorphous $\mathrm{SiO}_{2} / \mathrm{Si}$ substrate produced no straight etching lines, as seen in Figure 1g.

However, when we used r-plane sapphire the etching lines are aligned to a specific crystalline direction of the substrate thus producing parallel etching lines (Figure 1b,c). The SEM contrast changed depending on SEM magnification and condition, and in Figure 1c light and dark contrasts represent remaining graphene and underlying sapphire which appeared due to the graphene etching, respectively. The etching lines are straight and parallel to the [1101] 
direction of the sapphire substrate, signifying that the etching of graphene is guided by the surface anisotropy of the sapphire substrate and not by the graphene lattice. Under the same sputtering and etching conditions, SEM images show that graphene on r-plane of sapphire presents the highest density of etching lines with $\sim 20$ lines/ $\mu \mathrm{m}$ (Figure 1c). Additionally, these etching lines are straighter than those found in graphene supported on other substrates.

(a)
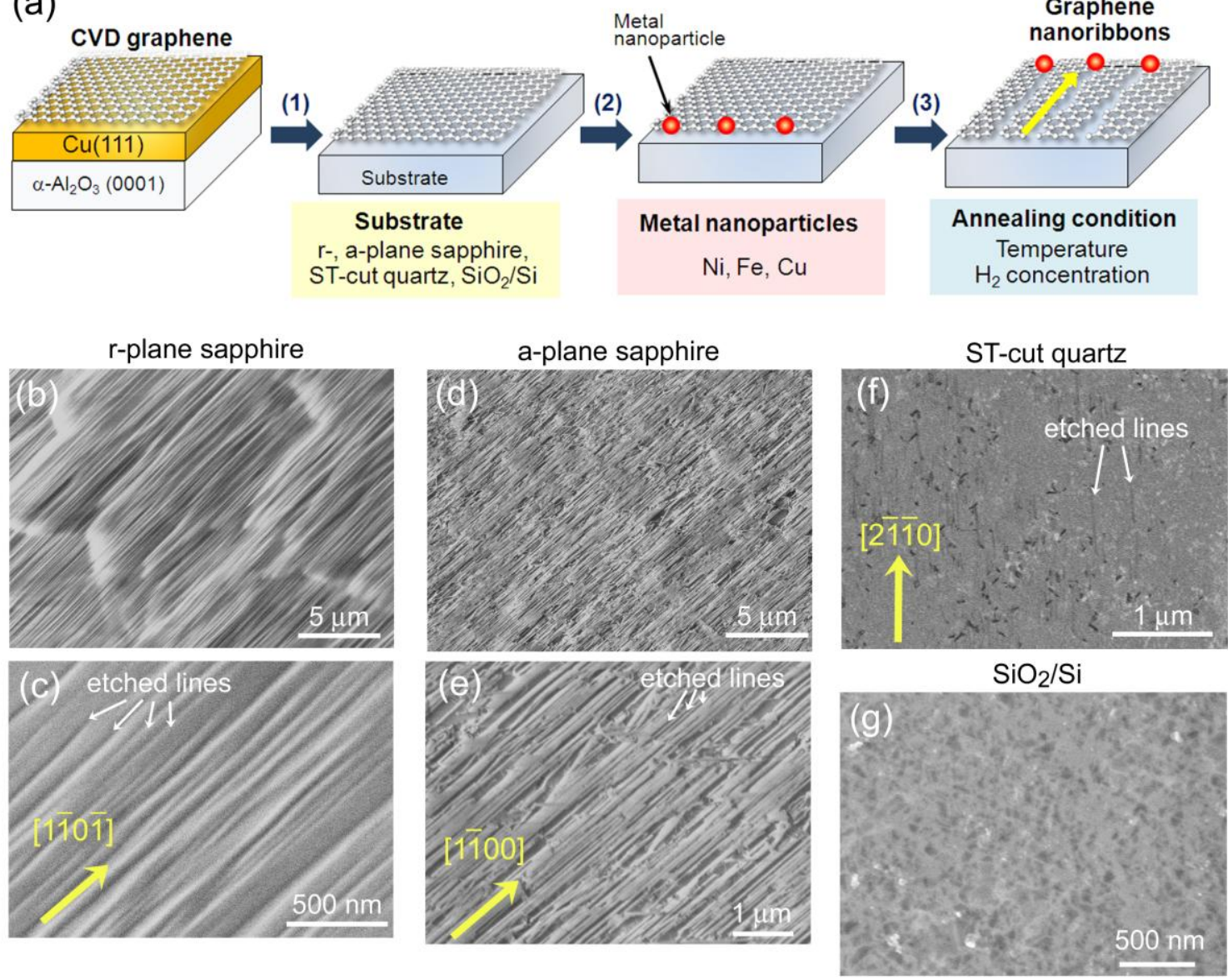

Fig. 1. (a) Schematic of graphene etching procedures: (1) Polymer-assisted transfer of CVD graphene to a target substrate, (2) Deposition of metal nanoparticles by magnetron sputtering, and (3) High temperature annealing in a $\mathrm{H}_{2} / \mathrm{Ar}$ mixed flow. SEM images of graphene annealed in the presence of Ni nanoparticles on sapphire r-plane (b,c), sapphire a-plane $(d, e)$, ST-cut quartz (f), and $\mathrm{SiO}_{2} / \mathrm{Si}$ (g) substrates. Etching temperature and $\mathrm{H}_{2}$ concentration are $1100{ }^{\circ} \mathrm{C}$ and $15 \%$, respectively. 
Figure 1d,e shows SEM images after etching of the CVD graphene on sapphire a-plane. The etching density was around 10 lines/ $\mu \mathrm{m}$ which is lower than that observed on sapphire $\mathrm{r}$ plane. Furthermore, although the lines are mainly parallel to the [1100] direction of sapphire a-plane, they often showed sudden and random changes of the direction. This decreases the yield of GNRs when compared with the case of etching on sapphire r-plane. In the case of the graphene etched on an ST-cut quartz substrate, the density of etched lines was very low (Figure 1f). Their length is also below one micron, while in the case of the two sapphire substrates lengths can go up to some tens of microns. Thus, under the present condition STcut quartz is comparatively an inadequate substrate for the production of GNRs.

The observed anisotropic graphene etching present many resemblances to the case of the CVD growth of horizontally-aligned single-walled carbon nanotubes (SWNTs) observed on crystalline sapphire and ST-cut quartz [46-51]. The orientations of the aligned SWNTs on rand a-plane sapphire are parallel to the [1101] and [1100] directions, respectively [46,47], which are consistent with those observed in the present metal-catalyzed etching of graphene. The horizontally-aligned SWNT growth was explained by the surface atomic arrangements of these sapphire surfaces; both sapphire $\mathrm{r}$ - and a-planes have anisotropic arrays of $\mathrm{Al}$ and $\mathrm{O}$ atoms along their respective in-plane directions, which guide the growing SWNTs [46-49]. Thus, we believe that the anisotropies on the sapphire surfaces are also responsible for guiding the movement of $\mathrm{Ni}$ nanoparticles, resulting in the aligned etching lines in graphene. The low degree of alignment on a-plane sapphire is ascribed to the lower degree of anisotropy compared with r-plane sapphire, which was also observed in the aligned SWNTs [47].

Unexpectedly, the graphene transferred on an ST-cut quartz substrate show few etching lines, which indicates that sapphire is a more efficient substrate for graphene etching. It is 
widely reported that highly aligned dense arrays of SWNTs can be also grown on ST-cut quartz [49-51], but the graphene etching occurs much less frequently than that on sapphire. The reason for this discrepancy is unclear, but we speculate that strong interaction or reaction between the $\mathrm{SiO}_{2}$ surface and the $\mathrm{Ni}$ nanoparticles prevents the movement of the $\mathrm{Ni}$ nanoparticles, since for example thermal diffusion of $\mathrm{Fe}$ nanoparticles into bulk $\mathrm{SiO}_{2}$ is known to occur even at $900{ }^{\circ} \mathrm{C}[53]$.

We note here that even in the absence of supporting substrates, the metal-assisted graphene etching can occur. Schäffel et al. demonstrated the etching of free-standing fewlayer graphene inside a transmission electron microscope (TEM) [37]. In this case, the etching direction is mainly determined by the graphene lattice, predominantly along zigzag directions. This result suggests that the few etched lines seen in the graphene deposited on ST-cut quartz and $\mathrm{SiO}_{2} / \mathrm{Si}$ substrates are probably due to the difficulty of the Ni nanoparticles to move on these substrates, reflecting a strong metal-support interaction and/or the absence of surface anisotropy.

\subsection{Effects of catalyst nature on the metal-assisted graphene etching}

To understand the influence of the metal catalyst on the graphene etching, three types of metal nanoparticles $(\mathrm{Ni}, \mathrm{Fe}$, and $\mathrm{Cu}$ ) were investigated. We used sapphire r-plane as a substrate, because it showed the best result among the studied substrates, while the etching temperature was fixed at $1100{ }^{\circ} \mathrm{C}$. As shown in Figure 2, metal species as well as $\mathrm{H}_{2}$ concentration strongly influenced the etching density of CVD grown graphene. It was observed that at either too high or low $\mathrm{H}_{2}$ concentrations, none of the catalysts was able to produce an appreciable quantity of etching lines. While for low $\mathrm{H}_{2}$ concentration $(7.5 \%)$ the etching reaction is probably limited by shortage of hydrogen (see eq. 1), for high concentration (50\%) large areas of graphene are etched away. 
Fe nanoparticles also produced a considerable amount of etching lines on the graphene, but the etching density was lower than for the case of the Ni nanoparticles (Figure 2c,e). Being different from $\mathrm{Ni}$ and $\mathrm{Fe}$ nanoparticles, $\mathrm{Cu}$ nanoparticles were found to be much less reactive toward the graphene etching with an etching density only $\sim 1$ line/ $\mu \mathrm{m}$. The low catalytic activity of $\mathrm{Cu}$ nanoparticles has also been reported for SWNT growth [54]. $\mathrm{Cu}$ nanoparticles are expected to agglomerate or evaporate easily at such high temperature (1100 $\left.{ }^{\circ} \mathrm{C}\right)$, considering the much lower melting temperature of elemental $\mathrm{Cu}\left(1084{ }^{\circ} \mathrm{C}\right)$ than $\mathrm{Ni}$ $\left(1455^{\circ} \mathrm{C}\right)$ and $\mathrm{Fe}\left(1536^{\circ} \mathrm{C}\right)$. This, along with the low carbon solubility in $\mathrm{Cu}$, renders $\mathrm{Cu}$ nanoparticles unsuitable for the etching of graphene.

It is difficult to understand why $\mathrm{Ni}$ nanoparticles show higher catalytic activity than $\mathrm{Fe}$ nanoparticles, as Fe is used much more frequently than Ni for SWNT growth. As we will show later the width of the etching lines is about $10 \mathrm{~nm}$, which is much wider than the diameter of metal nanoparticles used in the SWNT growth $(1-2 \mathrm{~nm})$. The catalytic decomposition of $\mathrm{CH}_{4}$ gas has been studied to produce $\mathrm{H}_{2}$ gas to be used as fuel, and it was reported that supported $\mathrm{Ni}$ nanoparticles are one of effective catalysts for this decomposition reaction $[55,56]$. We speculate that $\mathrm{Ni}$ nanoparticles tend to agglomerate at high temperatures which makes difficult to grow SWNTs with 1-2 nm diameters. However, since relatively large nanoparticles $(\sim 10 \mathrm{~nm})$ can contribute to the graphene etching, Ni nanoparticles exhibit high catalytic activity toward the metal-assisted etching, thus giving a high etching density. 


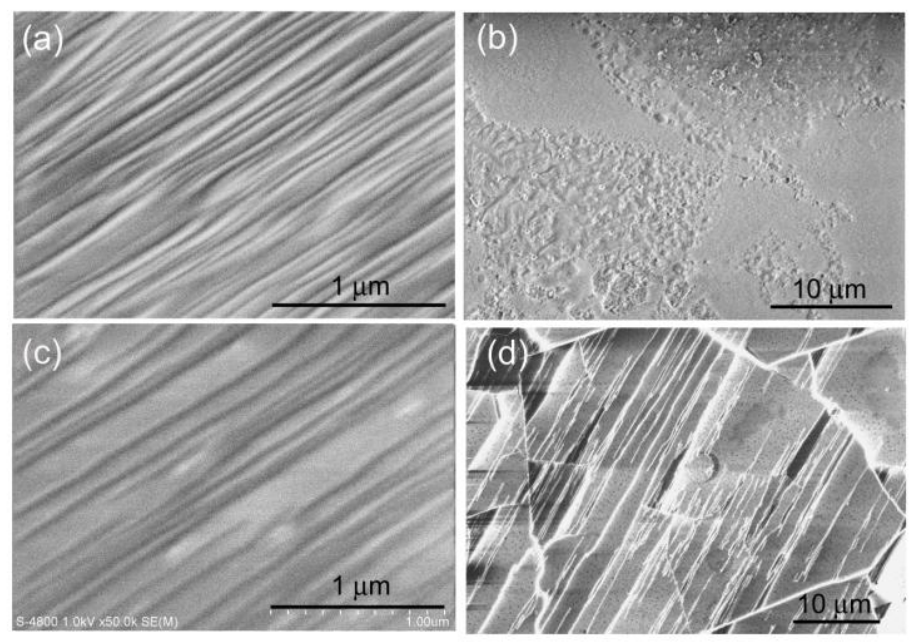

(e)

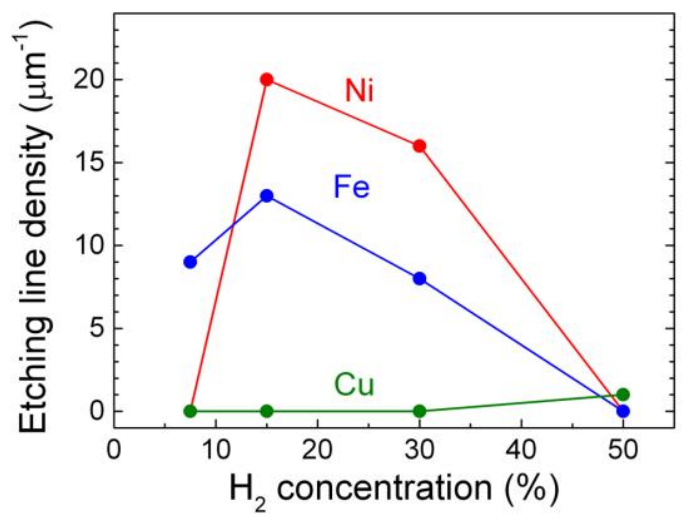

Fig. 2. SEM images of etched graphene with different metal nanoparticles and $\mathrm{H}_{2}$ concentrations. (a) $\mathrm{Ni}$ nanoparticles with $15 \% \mathrm{H}_{2}$, (b) $\mathrm{Ni}$ with $50 \% \mathrm{H}_{2}$, (c) $\mathrm{Fe}$ with $15 \% \mathrm{H}_{2}$, and (d) $\mathrm{Cu}$ with $50 \% \mathrm{H}_{2}$. Low magnification images are shown in (b,d) due to low etching density. (e) Hydrogen concentration dependence of etching density measured for $\mathrm{Ni}, \mathrm{Fe}$, and $\mathrm{Cu}$ nanoparticles.

\subsection{Temperature dependence}

We also studied how the etching density of Ni/graphene is affected by the etching temperature. When graphene was reacted with Ni nanoparticles at $900{ }^{\circ} \mathrm{C}$ almost no etching lines were observed (not shown here). Shown in Figure 3a-c is the evolution of the etching as temperature increases from 1000 to $1100{ }^{\circ} \mathrm{C}$ at a $\mathrm{H}_{2}$ concentration of $30 \%$. We found that at the lowest temperature, $1000{ }^{\circ} \mathrm{C}$, both the etching density and the degree of alignment are low (Figure 3a). At $1050{ }^{\circ} \mathrm{C}$ we observed a very high etching density of 26 lines/ $\mu \mathrm{m}$ with $30 \% \mathrm{H}_{2}$ gas, as seen in Figures $3 \mathrm{~b}$ and $3 \mathrm{~d}$. This is the highest density we found in the present work. The reaction at $1100{ }^{\circ} \mathrm{C}$ (Figure 3c) gave many straight lines but their density is lower than that reacted at $1050{ }^{\circ} \mathrm{C}$. 

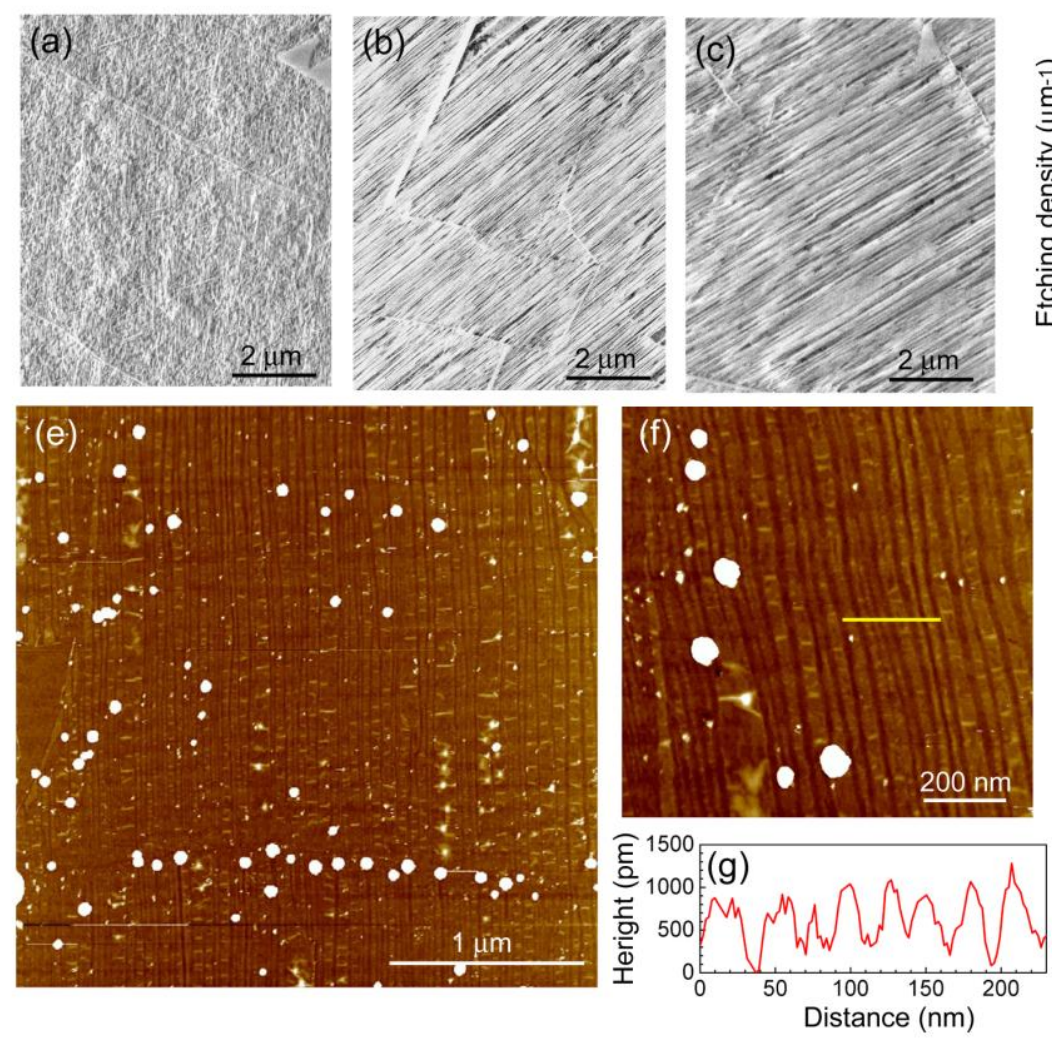
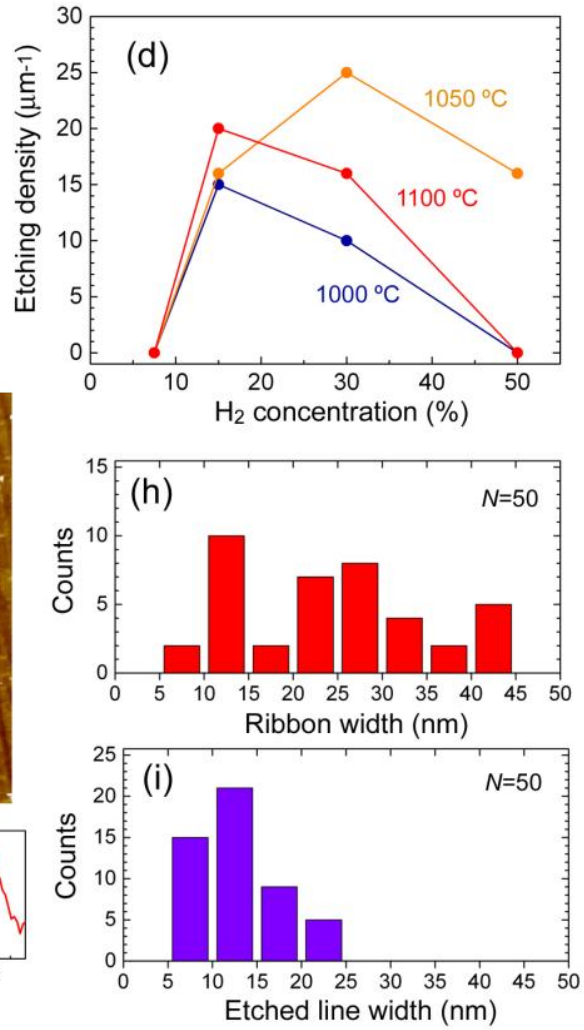

Fig. 3. SEM images of etched graphene at $1000{ }^{\circ} \mathrm{C}$ (a), $1050{ }^{\circ} \mathrm{C}$ (b), and $1100{ }^{\circ} \mathrm{C}$ (c) with $30 \% \mathrm{H}_{2}$ using Ni nanoparticles on sapphire r-plane. (d) Temperature dependence of etching densities measured under different $\mathrm{H}_{2}$ concentrations. (e,f) AFM images of etched graphene shown in (b). (g) Height profile measured for the yellow line in (f). Distribution of ribbon width (h) and etched line width (i) determined from AFM images.

It is interesting to note here that the highest density horizontally aligned SWNTs are grown at around $900{ }^{\circ} \mathrm{C}$ [45-52], but the present graphene etching showed the highest density at $1050{ }^{\circ} \mathrm{C}$. The higher temperature required for the graphene etching is probably due to the higher dissociation enthalpy of a C-C bond in graphene $(478 \mathrm{~kJ} / \mathrm{mol}$ for graphite; standard enthalpy of atomization of graphite $(716.7 \mathrm{~kJ} / \mathrm{mol})$ is divided by $3 / 2)$ than that of a $\mathrm{C}-\mathrm{H}$ bond in the $\mathrm{CH}_{4}$ feedstock $(435 \mathrm{~kJ} / \mathrm{mol})$ [57]. Thus, higher temperature is necessary for the 
continuous dissociation of $\mathrm{C}-\mathrm{C}$ bonds of a graphene sheet to realize long graphene etching lines. In addition, thermal agglomeration of metal nanoparticle catalysts needs to be suppressed in the SWNT growth, because small nanoparticles (1-2 nm) are necessary for the SWNT growth.

Corresponding AFM images for the optimum etching conditions are displayed in Figure 3e,f. Low magnification AFM image (Figure 3e) shows a large array of nanoribbons that was made by the metal-assisted etching. Large catalyst nanoparticles with diameters of 10-30 nm are widely observed (seen as white spots), which are supposed to form during the high temperature $\mathrm{H}_{2}$ annealing. High magnification AFM image (Figure 3f) and the corresponding height profile (Figure $3 \mathrm{~g}$ ) proves that the GNRs are composed of single-layer graphene, as the height difference $(\sim 0.8 \mathrm{~nm})$ matches the thickness of single-layer graphene observed by AFM in air $(0.9 \mathrm{~nm})$ [58]. In the AFM images, it can be appreciated that some nanoribbons have wrinkles normal to the etching direction probably due to strain relief during the cooling down in the $\mathrm{H}_{2}$ annealing process.

Figures $3 \mathrm{~h}$ and $3 \mathrm{i}$ show the width distributions of the remaining graphene (i.e., GNR width) and the etching lines, respectively, as determined from AFM images. The GNR width varied from 5 to $50 \mathrm{~nm}$ due to non-uniform etching density. Because the Ni nanoparticles were deposited by RF sputtering, the position of each nanoparticle was difficult to define precisely. The mean GNR width is $25 \mathrm{~nm}$, but it is expected that narrower ribbons can be obtained by further optimization of the $\mathrm{H}_{2}$ annealing and metal deposition processes. Interestingly, we found that the width distribution of the etching lines is more uniform than those of the remaining graphene nanoribbons width. The mean etching width is $10 \mathrm{~nm}$, and that is narrower than the mean width of GNRs. As the width of etching channels is closely related to the size of the catalyst particles, this result suggests that only Ni nanoparticles with very limited size are catalytically active towards graphene etching. 
The etching density of graphene is influenced by the density of the sputtered metal nanoparticles, as we reported previously [41]. AFM image of as-sputtered Ni nanoparticles on graphene surface is displayed in Supplementary data (Figure S1a). The initial nanoparticle size was 3-4 $\mathrm{nm}$ and it increased to 4-6 nm during the annealing in Ar at $400{ }^{\circ} \mathrm{C}$ (Figure $\mathrm{S} 1 \mathrm{~b}$ ). Further heating the substrate to etching temperature, $1000-1100{ }^{\circ} \mathrm{C}$ stimulates the thermal agglomeration of the nanoparticles, and the final nanoparticles react with graphene in the presence of hydrogen as expressed by eq. (1).

\subsection{Characterization of GNRs synthesized by metal-assisted etching}

The high density GNR arrays presented in Figure 3b were further characterized by Raman spectroscopy. Figure 4a shows the Raman spectrum of pristine graphene transferred on sapphire r-plane. The high 2D/G intensity ratio and an almost negligible D band verify that the starting graphene is high quality single-layer graphene. After the metal-assisted etching procedure, a sharp D band appears in the spectra, as seen in Figure 4b. Generally, the D band originates in defects, boundaries, wrinkles, and edges of graphene. The Raman spectra of the GNR array showed a clear polarization dependence; the D band intensity is high when the incident laser is polarized parallel to the direction of the nanoribbons, while D band is strongly suppressed when the laser is polarized normal to their direction. It is well known that the edges of graphene flakes show a clear polarization angle dependence of the D band, in contrast to other sources of the D band [59]. Therefore, we conclude the D band is mainly originated in the edges of the GNRs and not from defects of the graphene lattice, because there are a significant amount of graphene edges existing in the laser spot of our confocal Raman spectroscope (spot size $\sim 600 \mathrm{~nm}$ ). We also found that the $2 \mathrm{D}$ band remains more intense than $\mathrm{G}$ band even after the metal-assisted etching (see Figure $4 \mathrm{~b}$ ). In the case of GNRs produced by top-down methods using $\mathrm{O}_{2}$ plasma, Raman spectra show very weak 2D 
bands due to unavoidable damage to GNRs [28]. The high 2D/G intensity ratio observed in our GNR arrays indicates that the GNR array maintains a large part of the original high quality of the CVD graphene.
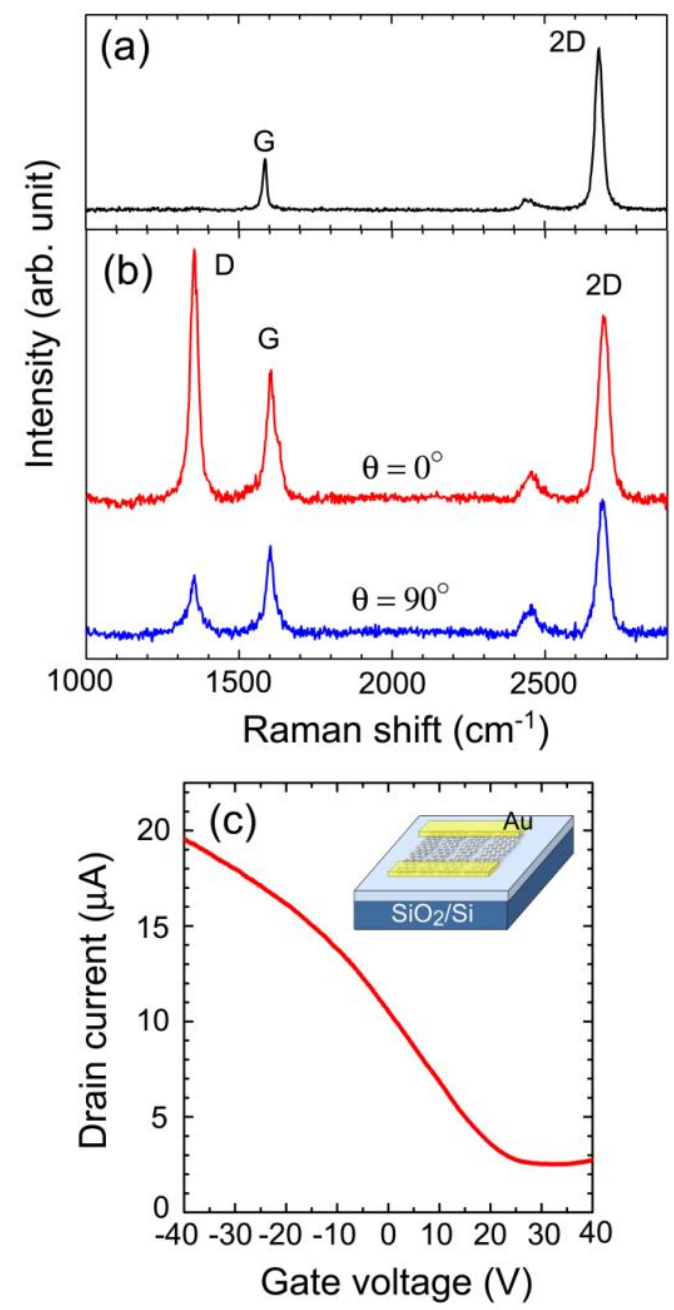

Fig. 4. (a) Raman spectrum of pristine CVD graphene before metal-assisted etching (measured on sapphire r-plane). (b) Polarized Raman spectra of a GNR array synthesized by anisotropic etching using Ni nanoparticles at $1050^{\circ} \mathrm{C}$. (b) Transfer characteristic of multichannel GNR device measured in vacuum at room temperature. The device was fabricated on $\mathrm{SiO}_{2} / \mathrm{Si}$ substrate after the re-transfer from sapphire r-plane. 
Finally, we measured the carrier transport properties of the GNR arrays synthesized by $\mathrm{Ni}$-assisted graphene etching. We transferred the arrays of GNRs onto a $\mathrm{SiO}_{2} / \mathrm{Si}$ substrate, and fabricated bottom-gate FETs using Au as electrodes. Figure 4c shows the transfer curve of the multi-channel GNR device. The channel width and length are set to $1 \mu \mathrm{m}$ and $2 \mu \mathrm{m}$, respectively. The on/off ratio measured within the gate voltage range $\left(V_{\mathrm{G}}= \pm 40 \mathrm{~V}\right)$ was $\sim 8$. Most probably the presence of wide ribbons dominate the current flow, thus lowering the on/off ratio, although narrow ribbons with width of less than $10 \mathrm{~nm}$ are present in the channel. Thus, further increase of uniformity of GNR width by improving the metal nanoparticle deposition and graphene etching processes is necessary. The transfer characteristic shows that the GNRs are highly p-type doped even though we measured in high vacuum $\left(\sim 10^{-4} \mathrm{~Pa}\right)$. It is likely that the GNR edges are mostly terminated with hydrogen, because the etching was performed in a flow of $\mathrm{H}_{2} / \mathrm{Ar}$ gas. The nanoribbon edges may also strongly bind to $\mathrm{O}_{2}$ and $\mathrm{H}_{2} \mathrm{O}$ molecules and/or resist, leading to the observed p-type behavior.

\section{Conclusions}

We demonstrate an approach to synthesize dense arrays of GNRs with controlled orientation by using metal-assisted etching on single-crystalline sapphire substrates. CVD grown single-layer graphene is used to produce large arrays of graphene nanoribbons. Our systematic study of the influence of the substrates, metal species, temperature, and $\mathrm{H}_{2}$ concentration enables the synthesis of high density GNRs. Under the optimized condition, we obtained the high etching density up to $\sim 26$ lines/ $\mu \mathrm{m}$ and narrow nanoribbons with an average width of $25 \mathrm{~nm}$. Raman spectroscopy showed that the GNRs maintain high quality. Our finding is expected to develop a novel method to prepare graphene nanoribbons in large scale without utilizing lithography processes. 


\section{Acknowledgments}

This work was supported by PRESTO-JST and JSPS Funding Program for Next Generation World-Leading Researchers (NEXT Program, \#GR075). P. S. -F. acknowledges the receipt of postdoctoral fellowship from JSPS. 


\section{REFERENCES}

[1] Geim AK, Novoselov KS. The rise of graphene. Nat Mater 2007;6:183-91.

[2] Avouris P, Graphene: electronic and photonic properties and devices. Nano Lett 2010;10:4285-94.

[3] Kim K, Choi JY, Kim T, Cho SH, Chung HJ. A role for graphene in silicon-based semiconductor devices. Nature 2011;479:338-44.

[4] Biswas C, Hee YH, Graphene versus carbon nanotubes in electronic devices. Adv Funct Mater 2011;21:3806-26.

[5] Bae S, Kim H, Lee Y, Xu X, Park JS, Zheng Y, et al. Roll-to-roll production of 30-inch graphene films for transparent electrodes. Nat Nanotechnol 2010;5:574-8.

[6] Yan C, Cho JH, Ahn, JH. Graphene-based flexible and stretchable thin film transistors. Nanoscale 2012;4:4870-82.

[7] Zhan D, Yan J, Lai L, Ni Z, Liu L, Shen Z. Engineering the electronic structure of graphene. Adv Mater 2012;24:4055-69.

[8] Oostinga JB, Heersche HB, Liu X, Morpurgo AF, Vandersypen LMK. Gate-induced insulating state in bilayer graphene devices. Nat Mater 2008;7:151-7.

[9] Zhang Y, Tang TT, Girit C, Hao Z, Martin MC, Zettl A., et al. Direct observation of a widely tunable bandgap in bilayer graphene. Nature 2009;459:820-3.

[10] Guinea, F.; Katsnelson, M. I.; Geim, A. K. Energy gaps and a zero-field quantum Hall effect in graphene by strain engineering. Nat Phys 2010;6:30-3.

[11] Loh KP, Bao Q, Ang PK, Yang, J. The chemistry of graphene. J Mater Chem 2010; 20:2277-89.

[12] Tanaka K, Yamashita S, Yamabe H, Yamabe T, Electronic properties of one-dimensional graphite family. Synth Met 1987;17:143-8. 
[13] Nakada K, Fujita M, Dresselhaus G, Dresselhaus MS. Edge state in graphene ribbons: nanometer size effect and edge shape dependence. Phys Rev B 1996;54:17954-61.

[14] Yang L, Park CH, Son YW, Cohen ML, Louie SG. Quasiparticle energies and band gaps in graphene nanoribbons. Phys Rev Lett 2007;99:186801.

[15] Ma L, Wang J, Ding F. Recent progress and challenges in graphene nanoribbon synthesis. Chem Phys Chem 2013;14:47-54.

[16] Cai J, Ruffieux P, Jaafar R, Bieri M, Braun T, Blankenburg S, et al. Atomically precise bottom-up fabrication of graphene nnoribbons. Nature 2010;466:470-3.

[17] Huang H, Wei D, Sun J, Wong SL, Feng YP, Castro Neto AH, et al. Spatially resolved electronic structures of atomically precise armchair graphene nanoribbons. Sci Rep 2012;2:983.

[18] Ago H, Ito Y, Tsuji M, Ikeda K. Step-templated CVD growth of aligned graphene nanoribbons supported by a single-layer graphene film. Nanoscale 2012;4:5178-82.

[19] Kato T, Hatakeyama R. Site- and alignment-controlled growth of graphene nanoribbons from nickel nanobars. Nat Nanotechnol 2012;7:651-6.

[20] Hayashi K, Sato S, Ikeda M, Kaneta C, Yokoyama N. Selective graphene formation on copper twin crystals. J Am Chem Soc 2012;134:12492-8.

[21] Ago H, Tanaka I, Ogawa Y, Yunus RM, Tsuji M, Hibino H. Lattice-oriented catalytic growth of graphene nanoribbons on heteroepitaxial nickel Films. ACS Nano 2013;7:1082533.

[22] Mattevi C, Kim H, Chhowalla MA. Review of chemical vapour deposition of graphene on copper. J Mater Chem 2011;21:3324-34.

[23] Ago H, Ogawa Y, Tsuji M, Mizuno S, Hibino H. Catalytic growth of graphene: toward large-area single-crystalline graphene. J Phys Chem Lett 2012;3:2228-36. 
[24] Yan M, Özylimaz B, Zhang Y, Kim P. Energy band-gap engineering of graphene nanoribbons. Phys Rev Lett 2007;98: 206805.

[25] Liang X, Wi S. Transport characteristics of multichannel transistors made from densely aligned sub-10 nm half-pitch graphene nanoribbons. ACS Nano 2012;6:9700-10.

[26] Stützel EU, Dufaux T, Sagar A, Rauschenbach S, Balasubramanian K, Burghard M, et al. Spatially resolved photocurrents in graphene nanoribbon devices. Appl Phys Lett 2013;102:43106.

[27] Pan Z, Liu N, Fu L, Liu Z. Wrinkle engineering: a new approach to massive graphene nanoribbon arrays. J Am Chem Soc 2011;133:17578-81.

[28] Ryu S, Maultzsch J, Han MY, Kim P, Brus LE. Raman spectroscopy of lithographically patterned graphene nanoribbons. ACS Nano 2011;5:4123-30.

[29] Jiao L, Zhang L, Wang X, Diankov G, Dai H. Narrow graphene nanoribbons from carbon nanotubes. Nature 2009;458:877-80.

[30] Kosynkin DV, Higginbotham AL, Sinitskii A, Lomeda JR, Dimiev A, Price BK, et al. Longitudinal unzipping of carbon nanotubes to form graphene nanoribbons. Nature 2009;458:872-76.

[31] Wang J, Ma L, Yuan Q, Zhu L, Ding F. Transition-metal-catalyzed unzipping of singlewalled carbon nanotubes into narrow graphene nanoribbons at low temperature. Ang Chem Int Ed 2011;50:8041-45.

[32] Huang CH, Su CY, Okada T, Li LJ, Ho KI, Li PW, et al. Ultra-low-edge-defect graphene nanoribbons patterned by neutral beam. Carbon 2013;61:229-35.

[33] Abbas AN, Liu G, Liu B, Zhang L, Liu H, Ohlberg D, et al. Patterning, characterization, and chemical sensing applications of graphene nanoribbon arrays down to $5 \mathrm{~nm}$ using helium ion beam lithography. ACS Nano 2014;8:1538-46. 
[34] Datta SS, Strachan DR, Khamis SM, Johnson ATC. Crystallographic etching of fewlayer graphene. Nano Lett 2008;8:1912-15.

[35] Ci L, Xu Z, Wang L, Gao W, Ding F, Kelly KF, et al. Controlled nanocutting of graphene. Nano Res 2008;1:116-22.

[36] Campos LC, Manfrinato VR, Sanchez-Yamagishi JD, Kong J, Jarillo-Herrero P. Anisotropic etching and nanoribbon formation in single-layer graphene. Nano Lett 2009;9:2600-04.

[37] Schäffel F, Wilson M, Bachmatiuk A, Rümmeli MH, Queitsch U, Rellinghaus B, et al. Atomic resolution imaging of the edges of catalytically etched suspended few-layer graphene. ACS Nano 2011;5:1975-83.

[38] Ma L, Wang J, Yip J, Ding F. Mechanism of transition-metal nanoparticle catalytic graphene cutting. J Phys Chem Lett 2014;5:1192-97.

[39] Tomita A, Tamai Y. An optical microscopic study on the catalytic hydrogenation of graphite. J Phys Chem 1974;78:2254-58.

[40] Takasu Y, Konishi S, Miyoshi R, Nukii K, Matsuse T, Sugimoto W, et al. Catalytic linear grooving of graphite surface layers by $\mathrm{Pt}, \mathrm{Ru}$, and $\mathrm{PtRu}$ nanoparticles. Chem Lett 2005;34:1008-9.

[41] Solís Fernández P, Yoshida K, Tsuji M, Ago H. Dense arrays of highly aligned graphene nanoribbons produced by substrate-controlled metal-assisted etching of graphene. Adv Mater $2013 ; 25: 6562-8$

[42] Tsukamoto T, Ogino T. Control of graphene etching by atomic structures of the supporting substrate surfaces. J Phys Chem C 2012;115:8580-5.

[43] Hu B, Ago H, Ito Y, Kawahara K, Tsuji M, Magome E, et al. Epitaxial growth of largearea single-layer graphene over $\mathrm{Cu}(111)$ /sapphire by atmospheric pressure CVD. Carbon 2012;50:57-65. 
[44] Orofeo CM, Hibino H, Kawahara K, Ogawa Y, Tsuji M, Ikeda K, et al. Influence of Cu metal on the domain structure and carrier mobility in single-layer graphene. Carbon 2012;50:2189-96.

[45] Ago H, Kawahara K, Ogawa Y, Tanoue S, Bissett MA, Tsuji M, et al. Epitaxial growth and electronic properties of large hexagonal graphene domains on $\mathrm{Cu}(111)$ thin film. Appl Phys Exp 2013;6:75101.

[46] Ago H, Nakamura K, Ikeda K, Uehara N, Ishigami N, Tsuji M. Aligned growth of isolated single-walled carbon nanotubes programmed by atomic arrangement of substrate surface. Chem Phys Lett 2005;408:433-38.

[47] Ago H, Uehara N, Ikeda K, Ohdo R, Nakamura K, Tsuji M. Synthesis of horizontallyaligned single-walled carbon nanotubes with controllable density on sapphire surface and polarized Raman spectroscopy. Chem Phys Lett 2006;421:399-403.

[48] Ishigami N, Ago H, Imamoto K, Tsuji M, Iakoubovskii K, Minami N. Crystal plane dependent growth of aligned single-walled carbon nanotubes on sapphire. J Am Chem Soc 2008;130:9918-24.

[49] Han S, Liu X, Zhou C. Template-free directional growth of single-walled carbon nanotubes on a- and r-plane sapphire. J Am Chem Soc 2005;127:5294-5.

[50] Kocabas C, Hur SH, Gaur A, Meitl MA, Shim M, Rogers JA. Guided growth of largescale, horizontally aligned arrays of single-walled carbon nanotubes and their use in thin-film transistors. Small 2005;1:1110-6.

[51] Inoue T, Hasegawa D, Badar S, Aikawa S, Chiashi S, Maruyama S. Effect of gas pressure on the density of horizontally aligned single-walled carbon nanotubes grown on quartz substrates. J Phys Chem C 2013;117:11804-10.

[52] Ding L, Yuan D, Liu J. Growth of high-density parallel arrays of long single-walled carbon nanotubes on quartz substrates. J Am Chem Soc 2008;130:5428-9. 
[53] Jung YJ, Homma Y, Ogino T, Kobayashi Y, Takagi D, Wei B, et al. High-density, largearea single-walled carbon nanotube networks on nanoscale patterned substrates. J Phys Chem B 2003;107:6859-64.

[54] Ago H, Nakamura Y, Ogawa Y, Tsuji M. Combinatorial catalyst approach for highdensity growth of horizontally aligned single-walled carbon nanotubes on sapphire. Carbon 2011;49:176-86.

[55] Ishihara T, Miyashita $\mathrm{Y}$, Isda $\mathrm{H}$, Takita Y. Decomposition of methane over $\mathrm{Ni} / \mathrm{SiO}_{2}$ catalysts with membrane reactor for the production of hydrogen. Chem Lett 1995:93-94.

[56] Takenaka S, Kobayashi S, Ogihara $\mathrm{H}$, Otsuka K. Ni/SiO${ }_{2}$ catalyst effective for methane decomposition into hydrogen and carbon nanofiber. J Catal 2003;217:79-87.

[57] Atkins PW. Physical Chemistry (5th edition), Oxford University Press, 1995.

[58] Ishigami M, Chen JH, Cullen WG, Fuhrer MS, Williams ED. Atomic Structure of Graphene on $\mathrm{SiO}_{2}$. Nano Lett 2007;7:1643.

[59] Cançado LG, Pimenta MA, Neves BRA, Dantas MSS, Jorio A. Influence of the atomic structure on the Raman spectra of graphite edges. Phys Rev Lett 2004;93:247401. 